PROCEEDINGS OF THE

AMERICAN MATHEMATICAL SOCIETY

Volume 129, Number 12, Pages 3623-3630

S 0002-9939(01)06011-7

Article electronically published on May 21, 2001

\title{
HARDY TYPE AND RELLICH TYPE INEQUALITIES ON THE HEISENBERG GROUP
}

\author{
PENGCHENG NIU, HUIQING ZHANG, AND YONG WANG
}

(Communicated by David S. Tartakoff)

\begin{abstract}
This paper contains some interesting Hardy type inequalities and Rellich type inequalities for the left invariant vector fields on the Heisenberg group.
\end{abstract}

\section{INTRODUCTION}

As is well known, Hardy's inequality and Rellich's inequality in Euclidean space $R^{n}$ (see [7], [9]) and their generalizations played important roles in many areas of mathematics. A natural and interesting question is: Can similar inequalities hold on the nilpotent Lie group, in particular, on the Heisenberg group $H_{n}$ ?

Recently Garofalo and Lanconelli [5] established the following Hardy type inequality:

$$
\int_{H_{n}}\left(\frac{|z|}{d}\right)^{2} \frac{|\Phi(x, y, t)|^{2}}{d^{2}} \leq\left(\frac{2}{Q-2}\right)^{2} \int_{H_{n}}\left|\nabla_{H_{n}} \Phi\right|^{2}, \forall \Phi \in C_{0}^{\infty}\left(H_{n} \backslash\{O\}\right)
$$

where $d$ denotes the Heisenberg distance: $d(x, y, t)=\left(|z|^{4}+t^{2}\right)^{\frac{1}{4}},|z|^{2}=x^{2}+y^{2}$, $z=(x, y) \in R^{n} \times R^{n}, t \in R, O=(0,0,0), Q$ the homogeneous dimension, $\nabla_{H_{n}} \Phi=$ $\left(X_{1} \Phi, \cdots, X_{n} \Phi, Y_{1} \Phi, \cdots, Y_{n} \Phi\right),\left\{X_{j}, Y_{j}\right\}_{j=1}^{n}$ the basis of left invariant vector fields on $H_{n}, X_{j}=\frac{\partial}{\partial x_{j}}+2 y_{j} \frac{\partial}{\partial t}, Y_{j}=\frac{\partial}{\partial y_{j}}-2 x_{j} \frac{\partial}{\partial t}$. Then they discuss some important topics including the unique continuation of the sub-Laplacian $\Delta_{H_{n}}=\sum_{j=1}^{n}\left(X_{j}^{2}+Y_{j}^{2}\right)$.

In this paper we give a general Hardy type inequality and Rellich type inequality on $H_{n}$. The methods here are based on the approach in Allegretto and Huang [3] for the $p$-Laplacian on $R^{n}$.

Theorem 1 (Hardy type inequality). Let $\Phi \in C_{0}^{\infty}\left(H_{n} \backslash\{O\}\right), 1<p<Q$. Then it follows that

$$
\int_{H_{n}}\left|\nabla_{H_{n}} \Phi\right|^{p} \geq\left(\frac{Q-p}{p}\right)^{p} \int_{H_{n}}\left(\frac{|z|}{d}\right)^{p} \frac{|\Phi|^{p}}{d^{p}}
$$

Received by the editors February 10, 2000 and, in revised form, April 20, 2000

2000 Mathematics Subject Classification. Primary 35H05, 46 E35.

Key words and phrases. Inequalities, left invariant vector fields, Heisenberg group.

This research was supported by the National Natural Science Foundation of China and the Shuangxin Plan at Northwestern Polytechnical University. 
and

$$
\int_{H_{n}}\left|\nabla_{H_{n}} \Phi\right|^{p} \geq\left(\frac{Q-p}{p}\right)^{p} \int_{H_{n}}\left(\frac{|z|}{d}\right)^{p} \frac{|\Phi|^{p}}{(1+d)^{p}} .
$$

Theorem 2 (Rellich type inequality). Let $\Phi \in C_{0}^{\infty}\left(H_{n} \backslash\{O\}\right), p \geq 1$. Then the inequality

$$
\int_{H_{n}}\left|\Delta_{H_{n}} \Phi\right|^{p}+C_{0} \int_{H_{n}} \frac{|z|^{2(p-2)}}{d^{4(p-1)}}|\Phi|^{p} \geq C_{1} \int_{H_{n}}\left(\frac{|z|^{2 p}}{d^{4 p}}\right)|\Phi|^{p}
$$

holds, where $C_{0}$ and $C_{1}$ only depend on $Q$ and $p$.

\section{Proof of Theorem 1}

We first deduce the Picone type identity for $\left\{X_{j}, Y_{j}\right\}$ which is especially useful for existence and nonexistence of $p$-sub-Laplace's equations and systems (for Laplace's equations and systems in $R^{n}$, see [3]).

Lemma 2.1 (Picone type identity). For differentiable functions $v>0, u \geq 0$ on $\Omega \subset H_{n}$, where $\Omega$ is a bounded or unbounded domain in $H_{n}$, or the whole space $H_{n}$, it holds that

$$
L(u, v)=R(u, v) \geq 0
$$

where

$$
\begin{gathered}
L(u, v)=\left|\nabla_{H_{n}} u\right|^{p}+(p-1) \frac{u^{p}}{v^{p}}\left|\nabla_{H_{n}} v\right|^{p}-p \frac{u^{p-1}}{v^{p-1}} \nabla_{H_{n}} u\left|\nabla_{H_{n}} v\right|^{p-2} \nabla_{H_{n}} v, \\
R(u, v)=\left|\nabla_{H_{n}} u\right|^{p}-\nabla_{H_{n}}\left(\frac{u^{p}}{v^{p-1}}\right)\left|\nabla_{H_{n}} v\right|^{p-2} \nabla_{H_{n}} v .
\end{gathered}
$$

Moreover, $L(u, v)=0$ a.e. on $\Omega$ iff $\nabla_{H_{n}}\left(\frac{u}{v}\right)=0$ a.e. on $\Omega$.

Proof. Since

$$
\begin{aligned}
& \nabla_{H_{n}}\left(\frac{u^{p}}{v^{p-1}}\right)=\frac{1}{v^{2 p-2}}\left[p u^{p-1} v^{p-1} \nabla_{H_{n}} u-(p-1) u^{p} v^{p-2} \nabla_{H_{n}} v\right], \\
& \nabla_{H_{n}}\left(\frac{u^{p}}{v^{p-1}}\right) \cdot \nabla_{H_{n}} v=p \frac{u^{p-1}}{v^{p-1}} \nabla_{H_{n}} u \cdot \nabla_{H_{n}} v-(p-1) \frac{u^{p}}{v^{p}}\left|\nabla_{H_{n}} v\right|^{2},
\end{aligned}
$$

it follows that (4) is obtained. On the other hand, $\frac{1}{p}+\frac{1}{q}=1$ and Young's inequality yield

$$
\begin{aligned}
L(u, v)= & \left|\nabla_{H_{n}} u\right|^{p}+(p-1) \frac{u^{p}}{v^{p}}\left|\nabla_{H_{n}} v\right|^{p}-p \frac{u^{p-1}}{v^{p-1}}\left|\nabla_{H_{n}} u\right| \cdot\left|\nabla_{H_{n}} v\right|^{p-1} \\
& +p \frac{u^{p-1}}{v^{p-1}}\left|\nabla_{H_{n}} v\right|^{p-2}\left(\left|\nabla_{H_{n}} u\right| \cdot\left|\nabla_{H_{n}} v\right|-\nabla_{H_{n}} u \cdot \nabla_{H_{n}} v\right) \\
= & p\left[\frac{\left|\nabla_{H_{n}} u\right|^{p}}{p}+\frac{\left(\frac{u}{v}\left|\nabla_{H_{n}} v\right|\right)^{q(p-1)}}{q}\right]-p \frac{u^{p-1}}{v^{p-1}}\left|\nabla_{H_{n}} u\right| \cdot\left|\nabla_{H_{n}} v\right|^{p-1} \\
& +p \frac{u^{p-1}}{v^{p-1}}\left|\nabla_{H_{n}} v\right|^{p-2}\left(\left|\nabla_{H_{n}} u\right| \cdot\left|\nabla_{H_{n}} v\right|-\nabla_{H_{n}} u \cdot \nabla_{H_{n}} v\right) \\
\geq & p \frac{u^{p-1}}{v^{p-1}}\left|\nabla_{H_{n}} v\right|^{p-2}\left(\left|\nabla_{H_{n}} u\right| \cdot\left|\nabla_{H_{n}} v\right|-\nabla_{H_{n}} u \cdot \nabla_{H_{n}} v\right) \geq 0
\end{aligned}
$$


and the equality holds if and only if $\left|\nabla_{H_{n}} u\right|=\frac{u}{v}\left|\nabla_{H_{n}} v\right|,\left|\nabla_{H_{n}} u\right|\left|\nabla_{H_{n}} v\right|=\nabla_{H_{n}} u$. $\nabla_{H_{n}} v$. Now suppose $L(u, v)\left(x_{0}\right)=0$. If $u\left(x_{0}\right) \neq 0$, then $\nabla_{H_{n}}\left(\frac{u}{v}\right)\left(x_{0}\right)=0$. If $u\left(x_{0}\right)=0$, then $\nabla_{H_{n}} u=0$ a.e. on $S=\{x \in \Omega \mid u(x)=0\}$ and $\nabla_{H_{n}}\left(\frac{u}{v}\right)=0$ a.e. on $S$. Therefore the statement is proved.

Remark 1. If the vector fields $\left\{X_{j}, Y_{j}\right\}$ are replaced by vector fields satisfying Hörmander's condition, then a similar identity is also valid.

It is clear that $\nabla_{H_{n}}\left(\frac{u}{v}\right)=0$ implies $u=k v$ for some constant $k$.

Let $S_{0}^{1, p}(\Omega)$ denote the completion of $C_{0}^{\infty}(\Omega)$ under the norm

$$
\|u\|_{S^{1, p}}=\left(\int_{\Omega}|u|^{p}+\left|\nabla_{H_{n}} u\right|^{p}\right)^{\frac{1}{p}}
$$

Theorem 2.1. Suppose that for some $\lambda>0, v \in C^{\infty}(\Omega)$ satisfies

$$
-\Delta_{H_{n}, p} v \geq \lambda g v^{p-1} \text { and } v>0 \text { in } \Omega,
$$

where $\Delta_{H_{n}, p}$ denotes the $p$-sub-Laplacian on $H_{n}$, i.e.

$$
\begin{aligned}
\Delta_{H_{n}, p}= & \sum_{j=1}^{n} X_{j}\left\{\left[\sum_{j=1}^{n}\left(\left|X_{j} v\right|^{2}+\left|Y_{j} v\right|^{2}\right)\right]^{\frac{p-2}{2}} X_{j} v\right\} \\
& +\sum_{j=1}^{n} Y_{j}\left\{\left[\sum_{j=1}^{n}\left(\left|X_{j} v\right|^{2}+\left|Y_{j} v\right|^{2}\right)\right]^{\frac{p-2}{2}} Y_{j} v\right\} .
\end{aligned}
$$

Then for any $u$ in $S_{0}^{1, p}$, it holds that

$$
\int_{\Omega}\left|\nabla_{H_{n}} u\right|^{p} \geq \lambda \int_{\Omega} g|u|^{p}
$$

Proof. Let $\Omega_{0} \subset \Omega, \Omega_{0}$ be compact. Take $\varphi \in C_{0}^{\infty}(\Omega), \varphi \geq 0$. By Lemma 2.1, we have

$$
\begin{aligned}
0 & \leq \int_{\Omega_{0}} L(\varphi, v) \leq \int_{\Omega} L(\varphi, v)=\int_{\Omega} R(\varphi, v) \\
& =\int_{\Omega}\left|\nabla_{H_{n}} \varphi\right|^{p}-\nabla_{H_{n}}\left(\frac{\varphi^{p}}{v^{p-1}}\right)\left|\nabla_{H_{n}} v\right|^{p-2} \nabla_{H_{n}} v \\
& =\int_{\Omega}\left(\left|\nabla_{H_{n}} \varphi\right|^{p}+\frac{\varphi^{p}}{v^{p-1}} \Delta_{H_{n}, p} v\right) \leq \int_{\Omega}\left(\left|\nabla_{H_{n}} \varphi\right|^{p}-\lambda g \varphi^{p}\right) .
\end{aligned}
$$

Let $\varphi \rightarrow u$ and (6) is easily obtained.

Proof of Theorem 1. Set $v=d^{\frac{p-Q}{p}}$. Since

$$
\nabla_{H_{n}} v=\left(\cdots, \frac{p-Q}{p} d^{-\frac{Q}{p}} X_{j} d, \cdots, \frac{p-Q}{p} d^{-\frac{Q}{p}} Y_{j} d, \cdots\right),
$$

then

$$
\left|\nabla_{H_{n}} v\right|=\frac{Q-p}{p} d^{-\frac{Q}{p}-1}|z|,\left|\nabla_{H_{n}} v\right|^{p-2}=\left(\frac{Q-p}{p}\right)^{p-2} d^{-\frac{(p+Q)(p-2)}{p}}|z|^{p-2},
$$


where we have used that $\left|\nabla_{H_{n}} d\right|=|z| d^{-1}$. Then

$$
\begin{aligned}
& -\Delta_{H_{n}, p} v=-\sum_{j=1}^{n}\left\{X_{j}\left[\left(\frac{Q-p}{p}\right)^{p-2} d^{-\frac{(p-2)(p+Q)}{p}}|z|^{p-2} X_{j} v\right]\right. \\
& \left.+Y_{j}\left[\left(\frac{Q-p}{p}\right)^{p-2} d^{-\frac{(p-2)(p+Q)}{p}}|z|^{p-2} Y_{j} v\right]\right\} \\
& =-\left(\frac{Q-p}{p}\right)^{p-2} \sum_{j=1}^{n}\left\{-\frac{(p-2)(p+Q)}{p} \cdot \frac{p-Q}{p} d^{-\frac{(p-2)(p+Q)}{p}-1-\frac{Q}{p}}\right. \\
& \cdot|z|^{p-2}\left(\left|X_{j} d\right|^{2}+\left|Y_{j} d\right|^{2}\right) \\
& +(p-2) \frac{p-Q}{p} \cdot d^{-\frac{(p-2)(p+Q)}{p}-\frac{Q}{p}}|z|^{p-4}\left(x_{j} X_{j} d+y_{j} Y_{j} d\right) \\
& +\frac{p-Q}{p} d^{-\frac{(p-2)(p+Q)}{p}}|z|^{p-2}\left[-\frac{Q}{p} d^{-\frac{Q}{p}-1}\left(\left|X_{j} d\right|^{2}+\left|Y_{j} d\right|^{2}\right)\right. \\
& \left.\left.+d^{-\frac{Q}{p}}\left(X_{j}^{2} d+Y_{j}^{2} d\right)\right]\right\} .
\end{aligned}
$$

Note that

$$
\sum_{j=1}^{n}\left(x_{j} X_{j} d+y_{j} Y_{j} d\right)=|z|^{4} d^{-3}, \Delta_{H_{n}} d=\sum_{j=1}^{n}\left(X_{j}^{2} d+Y_{j}^{2} d\right)=(Q-1)|z|^{2} d^{-3}
$$

and it follows that

$$
\begin{aligned}
-\Delta_{H_{n}, p} v= & \left(\frac{Q-p}{p}\right)^{p-1}\left[-\frac{(p-2)(p+Q)}{p}+(p-2)-\frac{Q}{p}+(Q-1)\right] \\
& \cdot|z|^{p} d^{\frac{-p^{2}-p Q+Q-p}{p}} \\
= & \left(\frac{Q-p}{p}\right)^{p} \frac{|z|^{p}}{d^{p}} d^{\frac{(p-Q)(p-1)}{p}-p}=\left(\frac{Q-p}{p}\right)^{p} \frac{|z|^{p}}{d^{p}} \frac{v^{p-1}}{d^{p}} .
\end{aligned}
$$

Inequality (1) is established by using Theorem 2.1 and inequality (2) is a consequence of (1).

Corollary 1 (Uncertainty principle). Suppose $u \in C_{0}^{\infty}\left(H_{n} \backslash\{O\}\right)$. Then

$$
\frac{Q-p}{p} \int_{H_{n}} \frac{|z|^{2}}{d^{2}}|u|^{2} \leq\left(\int_{H_{n}}\left|\nabla_{H_{n}} u\right|^{p}\right)^{\frac{1}{p}}\left(\int_{H_{n}}|z|^{q}|u|^{q}\right)^{\frac{1}{q}} .
$$

Proof. By (1) and Hölder's inequality, we get

$$
\begin{aligned}
\int_{H_{n}} \frac{|z|^{2}}{d^{2}}|u|^{2} & =\int_{H_{n}} \frac{|z||u|}{d^{2}} \cdot|z||u| \leq\left(\int_{H_{n}} \frac{|z|^{p}|u|^{p}}{d^{2 p}}\right)^{\frac{1}{p}}\left(\int_{H_{n}}|z|^{q}|u|^{q}\right)^{\frac{1}{q}} \\
& \leq \frac{p}{Q-p}\left(\int_{H_{n}}\left|\nabla_{H_{n}} u\right|^{p}\right)^{\frac{1}{p}}\left(\int_{H_{n}}|z|^{q}|u|^{q}\right)^{\frac{1}{q}} .
\end{aligned}
$$

Corollary 2. Suppose $u \in C_{0}^{\infty}\left(H_{n}\right)$. Then

$$
\int_{H_{n}} \frac{|z|^{p}|u|^{p}}{(1+d)^{2 p}} \leq\left(\frac{p}{Q-p}\right)^{p} \int_{H_{n}}\left|\nabla_{H_{n}} u\right|^{p} .
$$


Remark 2. Hardy type inequalities allow us to study the following eigenvalue problem in $H_{n}$ (if $p=2$, linear; if $p \neq 2$, nonlinear) with indefinite weights

$$
\begin{aligned}
-\Delta_{H_{n}, p} u & =\lambda g|u|^{p-2} u, & & \text { in } H_{n}, \\
u & \rightarrow 0, & & \text { as } d(x, y, t) \rightarrow \infty
\end{aligned}
$$

(see Allegretto [1, Allegretto and Huang [2], and Huang [8] for the Laplacian or $p$ Laplacian case in $R^{n}$ ).

Remark 3 . The above inequalities are also applied to the study of unique continuation for $\Delta_{H_{n}, p} ;$ see [6], [5].

\section{Proof of Theorem 2}

Lemma 3.1. If $v$ is a smooth function satisfying

$$
v>0, \Delta_{H_{n}} v<0, \text { in } \Omega,
$$

and $u \geq 0, p>1$, then it follows that

$$
L_{1}(u, v)=R_{1}(u, v) \geq 0,
$$

where

$$
\begin{gathered}
L_{1}(u, v)=\left|\Delta_{H_{n}} u\right|^{p}-p \frac{u^{p-1}}{v^{p-1}} \Delta_{H_{n}} u \cdot \Delta_{H_{n}} v\left|\Delta_{H_{n}} v\right|^{p-2}+(p-1) \frac{u^{p}}{v^{p}}\left|\Delta_{H_{n}} v\right|^{p} \\
-p(p-1) \frac{u^{p-2}}{v^{p-1}}\left|\Delta_{H_{n}} v\right|^{p-2} \Delta_{H_{n}} v \\
\cdot\left(\left|\nabla_{H_{n}} u\right|^{2}-2 \frac{u}{v} \nabla_{H_{n}} u \cdot \nabla_{H_{n}} v+\frac{u^{2}}{v^{2}}\left|\nabla_{H_{n}} v\right|^{2}\right), \\
R_{1}(u, v)=\left|\Delta_{H_{n}} u\right|^{p}-\Delta_{H_{n}}\left(\frac{u^{p}}{v^{p-1}}\right)\left|\Delta_{H_{n}} v\right|^{p-2} \Delta_{H_{n}} v .
\end{gathered}
$$

Proof. Note that

$$
\begin{aligned}
\Delta_{H_{n}}\left(\frac{u^{p}}{v^{p-1}}\right)= & \frac{1}{v^{2 p-2}}\left[p(p-1) u^{p-2}\left|\nabla_{H_{n}} u\right|^{2} v^{p-1}+p u^{p-1} v^{p-1} \Delta_{H_{n}} u\right. \\
& -2 p(p-1) u^{p-1} v^{p-2} \nabla_{H_{n}} u \cdot \nabla_{H_{n}} v-(p-1)(p-2) u^{p} v^{p-3}\left|\nabla_{H_{n}} v\right|^{2} \\
& \left.-(p-1) u^{p} v^{p-2} \Delta_{H_{n}} v\right] \\
& +\frac{2(p-1)^{2}}{v^{p+1}} u^{p}\left|\nabla_{H_{n}} v\right|^{2}
\end{aligned}
$$

and (9) is evidently obtained.

Since $\Delta_{H_{n}} v<0$ and

$$
\frac{u^{p-1}}{v^{p-1}} \Delta_{H_{n}} u \Delta_{H_{n}} v\left|\Delta_{H_{n}} v\right|^{p-2} \leq \frac{\left|\Delta_{H_{n}} u\right|^{p}}{p}+\frac{1}{q} \frac{u^{p}}{v^{p}}\left|\Delta_{H_{n}} v\right|^{p}, \frac{1}{p}+\frac{1}{q}=1,
$$


we have

$$
\begin{aligned}
L_{1}(u, v) \geq & \left|\Delta_{H_{n}} u\right|^{p}+(p-1) \frac{u^{p}}{v^{p}}\left|\Delta_{H_{n}} v\right|^{p}-p\left(\frac{\left|\Delta_{H_{n}} u\right|^{p}}{p}+\frac{1}{q} \frac{u^{p}}{v^{p}}\left|\Delta_{H_{n}} v\right|^{p}\right) \\
& -p(p-1) \frac{u^{p-2}}{v^{p-1}}\left|\Delta_{H_{n}} v\right|^{p-2} \Delta_{H_{n}} v\left|\nabla_{H_{n}} u-\frac{u}{v} \nabla_{H_{n}} v\right|^{2} \\
= & \left(p-1-\frac{p}{q}\right) \frac{u^{p}}{v^{p}}\left|\Delta_{H_{n}} v\right|^{p}-p(p-1) \frac{u^{p-2}}{v^{p-1}}\left|\Delta_{H_{n}} v\right|^{p-2} \Delta_{H_{n}} v \\
& \cdot\left|\nabla_{H_{n}} u-\frac{u}{v} \nabla_{H_{n}} v\right|^{2} \\
\geq & 0 .
\end{aligned}
$$

Theorem 3.1. Let $v \in C^{\infty}(\Omega), v>0$, satisfying

$$
\Delta_{H_{n}}\left(\left|\Delta_{H_{n}} v\right|^{p-2} \Delta_{H_{n}} v\right) \geq-\lambda g_{1} v^{p-1}+\mu g_{2} v^{p-1}
$$

for some constant $\lambda, \mu>0, \Delta_{H_{n}} v<0$. Then for any $u \in S_{0}^{2, p}(\Omega)$, it holds that

$$
\int_{\Omega}\left|\Delta_{H_{n}} u\right|^{p}+\lambda \int_{\Omega} g_{1}|u|^{p} \geq \mu \int_{\Omega} g_{2}|u|^{p}
$$

where $S_{0}^{2, p}(\Omega)$ is the completion of $C_{0}^{\infty}(\Omega)$ in the Folland-Stein space $S^{2, p}(\Omega)$ (see [4]).

Proof. Suppose $\Omega_{0} \subset \Omega, \Omega_{0}$ is compact. Take $\varphi \in C_{0}^{\infty}(\Omega), \varphi \geq 0$. It follows that

$$
\begin{aligned}
0 & \leq \int_{\Omega_{0}} L_{1}(\varphi, v) \leq \int_{\Omega} L_{1}(\varphi, v)=\int_{\Omega} R_{1}(\varphi, v) \\
& =\int_{\Omega}\left|\Delta_{H_{n}} \varphi\right|^{p}-\int_{\Omega} \Delta_{H_{n}}\left(\frac{\varphi^{p}}{v^{p-1}}\right)\left|\Delta_{H_{n}} v\right|^{p-2} \Delta_{H_{n}} v \\
& =\int_{\Omega}\left|\Delta_{H_{n}} \varphi\right|^{p}-\int_{\Omega} \frac{\varphi^{p}}{v^{p-1}} \Delta_{H_{n}}\left(\left|\Delta_{H_{n}} v\right|^{p-2} \Delta_{H_{n}} v\right) \\
& \leq \int_{\Omega}\left|\Delta_{H_{n}} \varphi\right|^{p}+\lambda \int_{\Omega} g_{1} \varphi^{p}-\mu \int_{\Omega} g_{2} \varphi^{p} .
\end{aligned}
$$

Letting $\varphi \rightarrow u$, we prove the result.

Proof of Theorem 2. Set $v=d^{\beta}$, where $\beta<0$ will be determined later. It is clear that

$$
\Delta_{H_{n}} v=\beta(\beta+Q-2)|z|^{2} d^{\beta-4} .
$$


Letting $\beta>2-Q$ yields $\Delta_{H_{n}} v<0$. A direct calculation gives

$$
\begin{aligned}
\Delta_{H_{n}}\left(\left|\Delta_{H_{n}} v\right|^{p-2} \Delta_{H_{n}} v\right)= & \beta|\beta|^{p-2}(\beta+Q-2)^{p-1} \Delta_{H_{n}}\left[|z|^{2(p-1)} d^{(\beta-4)(p-1)}\right] \\
= & \beta|\beta|^{p-2}(\beta+Q-2)^{p-1}\left\{4 n(p-1)|z|^{2(p-2)} d^{(\beta-4)(p-1)}\right. \\
+ & 4(p-1)(p-2)|z|^{2(p-2)} d^{(\beta-4)(p-1)} \\
+ & 4(p-1)^{2}(\beta-4)|z|^{2(p-2)} d^{(\beta-4)(p-1)-1} \\
& \cdot \sum_{j=1}^{n}\left(x_{j} X_{j} d+y_{j} Y_{j} d\right) \\
+ & (p-1)(\beta-4)[(\beta-4)(p-1)-1] \\
& \cdot|z|^{2(p-1)} d^{(\beta-4)(p-1)-2}\left|\nabla_{H_{n}} d\right|^{2} \\
+ & \left.(p-1)(\beta-4)|z|^{2(p-1)} d^{(\beta-4)(p-1)-1} \Delta_{H_{n}} d\right\} \\
= & \beta|\beta|^{p-2}(\beta+Q-2)^{p-1}(p-1)\{4(n+p-2) \\
& \cdot|z|^{2(p-2)} d^{(\beta-4)(p-1)} \\
+ & (\beta-4)[4(p-1)+(\beta-4)(p-1)+Q-2] \\
& \left.\cdot|z|^{2 p} d^{(\beta-4)(p-1)-4}\right\}
\end{aligned}
$$

where we have used the identities $\sum_{j=1}^{n}\left(x_{j} X_{j} d+y_{j} Y_{j} d\right)=|z|^{4} d^{-3},\left|\nabla_{H_{n}} d\right|^{2}=|z|^{2} d^{-2}$, $\Delta_{H_{n}} d=(Q-1)|z|^{2} d^{-3}$.

We take $\beta$ satisfying $\max \left(\frac{2-Q}{p-1}, \frac{-(n-2 p+2)-\sqrt{n^{2}+4 p-4}}{p-1}\right)<\beta<0$ and denote

$$
\begin{gathered}
C_{0}=-4 \beta|\beta|^{p-2}(\beta+Q-2)^{p-1}(p-1)(n+p-2)>0, \\
C_{1}=\beta|\beta|^{p-2}(\beta+Q-2)^{p-1}(p-1)(\beta-4)[\beta(p-1)+Q-2]>0 .
\end{gathered}
$$

It follows that $C_{1}>C_{0}$ and

$$
\Delta_{H_{n}}\left(\left|\Delta_{H_{n}} v\right|^{p-2} \Delta_{H_{n}} v\right) \geq-C_{0} \frac{|z|^{2(p-2)}}{d^{4(p-1)}} v^{p-1}+C_{1} \frac{|z|^{2 p}}{d^{4 p}} v^{p-1} .
$$

By Theorem 3.1, (3) is deduced.

\section{ACKNOWLEDGEMENT}

The authors would like to thank Professor Luo Xuebo for helpful discussions. We would like to thank the referee for his careful reading of the paper and for several valuable comments.

\section{REFERENCES}

[1] Allegretto, W., Principal eigenvalues for indefinite weight elliptic problem in $R^{n}$, Proc. Amer. Math. Soc., 116 (1992), 701-706. MR 93a:35114

[2] Allegretto, W. and Huang, Y.X., Eigenvalues of the indefinite weight $p$-Laplacian in weighted spaces, Funk. Ekv. 38 (1995), 233-242. MR 96j:35184

[3] Allegretto, W. and Huang, Y.X., A Picone's identity for the p-Laplacian and applications, Nonlinear Anal. 32 (1998), 819-830. MR 99c:35051

[4] Folland, G.B. and Stein, E.M., Estimates for the $\overline{\partial_{b}}$-complex and analysis on the Heisenberg group, Comm. Pure Appl. Math., 27 (1974), 429-522. MR 51:3719 
[5] Garofalo, N. and Lanconelli, E., Frequency functions on the Heisenberg group, the uncertainty principle and unique continuation, Ann. Inst. Fourier, Grenoble, 40 (1990), 313-356. MR 91i:22014

[6] Garofalo,N. and Lin, F.H., Unique continuation for elliptic operators: A geometric-variational approach, Comm. Pure Appl. Math., 40 (1987), 347-366. MR 88j:35046

[7] Hardy,G.H., Littlewood, J.E. and Polya, G., Inequalities, Cambridge Univ. Press, 2nd ed., 1952. MR 13:727

[8] Huang, Y.X., Eigenvalues of the $p$-Laplacian in $R^{n}$ with indefinite weight, Comment. Math. Univ. Carolinae 36 (1995), 519-527. MR 96h:35063

[9] Rellich,F., Perturbation theory of eigenvalue problems, Gordon and Breach, New York, 1969. MR 39:2014

Department of Applied Mathematics, Northwestern Polytechnical University, Xi'an, 710072, People's Republic of China

E-mail address: niupengcheng.iamlxl@nwpu.edu.cn

Department of Applied Mathematics, Northwestern Polytechnical University, Xi'an, 710072, People's Republic of China

Department of Science and Engineering of Management, Sichuan University, Chengdu, 610064, People's Republic of China 